\title{
Bone health and evaluation of bone mineral density in patients with premature ovarian insufficiency
}

\author{
Anna Szeliga, Marzena Maciejewska-Jeske, Błażej Męczekalski \\ Department of Gynaecological Endocrinology, Poznan University of Medical Sciences, Poznan, Poland
}

\begin{abstract}
Oestrogens exert an influence on skeletal homeostasis during growth and adulthood. Regulation of osteoclasts and osteoblasts generation and apoptosis and prolongation of the lifespan of osteocytes are some of their actions on bone metabolism. Premature ovarian insufficiency (POI) and associated loss of oestrogen action on osteoclasts leads to trabecular perforation and loss of connectivity. Lack of oestrogens acting on osteoblast progenitors also causes a decrease in critical bone mass. Postmenopausal hypoestrogenism is associated with an increase in the number of lymphocyte B-cells expressing nuclear factor $\kappa B$ ligand (RANKL) in the bone marrow and elevated expression of RANKL by B-cells. Increased concentration of RANKL stimulates activation of osteoclasts and leads to oestrogen deficiency-associated bone loss. It has been proven that women with POI have decreased bone mineral density (BMD) measured in lumbar spine and femoral neck. The loss of bone mass associated with oestrogen deficiency is greater in trabecular than in cortical bone, thus women with POI have a significant decrease in BMD, particularly in the lumbar spine vertebrae. Smoking cessation, weight-bearing, and muscle-strengthening exercises on most days of the week, avoidance of excessive alcohol intake, and adequate supplementation of calcium and vitamin $\mathrm{D}$ are the main lifestyle rules necessary to avoid decline in BMD. The most important component of decreased BMD treatment in POI patients is systemic hormonal replacement therapy (HRT). HRT should provide hormonal balance and should mimic normal ovarian function as much as possible.
\end{abstract}

Key words: premature ovarian insufficiency, early menopause, hormonal replacement therapy, bone mineral density, osteoporosis, osteopaenia.

\section{Introduction}

Oestrogens and androgens exert an influence on skeletal homeostasis during growth and adulthood. An increase in production of growth factors, enhanced response to mechanical strains, regulation of osteoclasts and osteoblasts generation and apoptosis, epiphyseal closure, and prolongation of the lifespan of osteocytes are some of their actions on bone metabolism [1]. Postmenopausal decline in oestrogen concentration is a well-known factor causing a decrease in bone mass [2]. Nonetheless, significant bone loss starts immediately after achieving peak bone mass regardless of changes in sex steroid concentrations. After menopause, due to a decrease in oestradiol concentration, loss of bone density accelerates. It achieves a similar rate as in eugonadal men in the same age independently of sex hormones within 10 years after menopause [3].

\section{Bone loss due to oestrogen deficiency}

At the beginning of menopause loss of oestrogen action on osteoclasts leads to trabecular perforation and loss of connectivity. A lack of oestrogens acting on osteoblast progenitors causes also a decrease in critical bone mass [4]. After a few years of acceleration, a slow phase of decline in bone wall width starts. It is associated mainly with age and caused particularly by insufficient supply of osteoblasts [5]. Decline in physical activity, decreased muscle mass, and insufficient production of growth factors appear to be the principal pathophysiological pathway for the involution of bone mass associated with age. Additionally, increased cortical osteocyte apoptosis contributes to increased secretion of nuclear factor $\kappa \mathrm{B}$ ligand (RANKL) by other healthy osteocytes, and decreased vascularity and nutrition of bone. It has been established that after 65 years of age loss of bone mass appears mainly due to loss of cortical bone and increased intracortical porosity. The presence of these processes corresponds with an increase in non-vertebral fractures in individuals aged $>65$ years. Nevertheless, the latest trials suggest that oestrogen deficiency can be also associated with bone turnover in late postmenopausal women due to extraskeletal effects of oestrogens, like intestinal calcium absorption, renal calcium handling, and direct effects on parathormone secretion [6]. 


\section{The influence of RANKL on bone loss}

Target cells receive signals from sex steroids by nuclear hormone receptor family: oestrogen receptor (ER) $\alpha$ or ER $\beta$, and androgen receptor (AR). Those receptors are zinc finger-containing transcription factors. During menopause the decreased concentration of oestrogens contributes to loss of both the trabecular and cortical bone compartments. The decline in oestrogen concentration leads to an increase in the bone remodelling rate with elevation of both osteoblasts and osteoclasts numbers. Nevertheless, bone matrix removal mediated by osteoclasts is much higher than osteoblast bone formation, leading to bone loss [7]. Resorption of bone is mediated by increased activity of the RANKL acting on RANK receptors on osteoclasts and their precursors. After menopause, due to oestrogen deficiency, the number of lymphocyte B-cells expressing RANKL in the bone marrow increases. Noteworthy is the fact that it is not estimated whether the increase in the total number of B-cells or elevated expression of RANKL per cell stimulates activation of osteoclast and leads to oestrogen deficiency-associated bone loss. It is also noticed that menopause is associated with higher production of RANKL by T-cells; nevertheless, it seems that an increase in T-cell production of RANKL is not required for postmenopausal bone loss [8].

\section{Bone mineral density in premature ovarian insufficiency patients}

It has been established that $90 \%$ of peak bone mass is achieved until the age of 18 years. Therefore, oestrogen deficiency in women suffering from premature ovarian insufficiency (POI) has a significant negative impact on bone mineral density (BMD), particularly because oestrogen deficiency may appear in the phase of intensive gain in bone mass [9]. The effect of POI-associated oestrogen deficiency on BMD is well established. Women with $\mathrm{POI}$ have decreased BMD assessed by dual-energy X-ray absorptiometry (DEXA) of the lumbar spine and femoral neck. The loss of bone associated with oestrogen deficiency is greater in trabecular than in cortical bone, thus women with POI have a significant decrease in BMD, particularly in the vertebrae of the lumbar spine. Despite the pathological mechanism of POI, Turner syndrome, other gonadal dysgeneses, chemotherapy, or idiopathic POI, women suffering from POI have decreased BMD [10-12]. Previous studies indicated that 60 of 89 karyotypically normal women with spontaneous POI had significantly decreased BMD measured in the femoral neck when compared to healthy controls. Forty-seven per cent of POI patients diagnosed within 1.5 years had significantly decreased BMD as well [13]. In another study POI patients had significantly decreased BMD measured in the lower femoral neck when compared with control women $\left(0.77\right.$ vs. $0.81 \mathrm{~g} / \mathrm{cm}^{2}$, respectively; $p=0.001$ ) [11]. In another study, $15 \%$ of POI patients had a Z-score below -2 ( $p=0.005$ when compared to controls) [14]. Similarly, in another trial a significant decrease in BMD of the femur neck $(0.715$ vs. $0.762 ; p<0.05)$ and lumbar spine (0.913 vs. 0.985 ; $p<0.05)$ of POI patients when compared to healthy controls was described [15].

It is well established that Turner syndrome is commonly associated with POI. Recent studies conducted on patients suffering from POI due to Turner syndrome revealed that $30.7 \%$ of patients had a medical history of trauma-associated fractures, 32 from 43 previously diagnosed with $\mathrm{POI}$ had already diagnosed abnormal $B M D$, and the next 64 from 111 patients who underwent BMD measurements during the study were diagnosed with osteoporosis and osteopenia (8 and 56 patients, respectively) [10].

Chemotherapy in young age can contribute to POI development, thus decreasing BMD. POI patients after chemotherapy due to gynaecological malignancies had significantly decreased BMD when compared to controls (39\% vs. $15 \%$, respectively; $p=0.009$ ) [16]. Particularly important is the fact that women who underwent chemotherapy did not suffer from BMD deprivation immediately after treatment, but after more than 18 months. This observation indicates that not chemotherapy but decreased concentration of oestrogens due to $\mathrm{POI}$ is the reason for the BMD decrease [12].

The most important risk factors contributing to BMD loss in POI are degree and duration of oestrogen deficiency as well as oestrogen replacement nonadherence [17]. The additional risk factor for decreased BMD in POI is early diagnosis of POI, more than a one-year delay in diagnosis of POI, concentration of vitamin D below $32 \mathrm{ng} / \mathrm{ml}$, low calcium intake, and lack of physical exercise [14, 18].

\section{Assessment of bone mineral density}

The gold standard of BMD assessment is DEXA of the lumbar spine (vertebrae L1-L4), hip (femoral neck, total hip), or forearm (1/3 distal radius). Measurements are presented as mean peak BMD and SD either as T-score or Z-score. The T-score is calculated from the patient's current BMD and young (30-year-old) normal reference mean. Conversely, the Z-score is calculated from the patient's BMD and age-matched normal BMD. The Z-score is used to present BMD in younger patients or those suffering from severe osteoporosis. International guidelines indicate that osteoporosis can be diagnosed when the T-score of BMD at the lumbar spine or femoral neck is below $-2.5 \mathrm{SD}$, and osteopaenia when $-2.5 \mathrm{SD}<\mathrm{T}$-score $<-1.0$ SD [16]. The first measurement of BMD should be taken at the moment of diagnosis of POI. Notwithstanding the fact that DEXA remains a gold standard of BMD 
assessment, additional methods such as quantitative computed tomography or quantitative ultrasound are potentially useful [19]. The fracture risk assessment tool (FRAX) is a helpful tool for assessment of bone fracture risk. Nevertheless, it is useful in patients between 40 and 90 years old, and thus it is difficult to apply it for POI patients.

\section{Treatment}

Non-pharmacological strategies and lifestyle changes are first-line interventions in the case of decreased BMD. The National Osteoporosis Foundation strongly recommends several methods, which are primary goals in maintaining bone health. Smoking cessation, weight-bearing and muscle-strengthening exercises on most days of the week, avoidance of excessive alcohol intake, and adequate supplementation of calcium and vitamin D are the main lifestyle rules [20]. WHO guidelines indicate that $1000 \mathrm{mg}$ /day of calcium intake is sufficient for maintaining proper BMD, while taking more than 1200 to 1500 mg of calcium per day may increase the risk of developing kidney stones or cardiovascular disease and has no positive effect on bone mass. 800 IU of vitamin D seems to be an adequate daily intake. Nevertheless, supplementation of vitamin $\mathrm{D}$ should be prescribed under $25(\mathrm{OH}) \mathrm{D}$ control (recommended serum concentration is higher than $30 \mathrm{ng} / \mathrm{ml})[17,20]$.

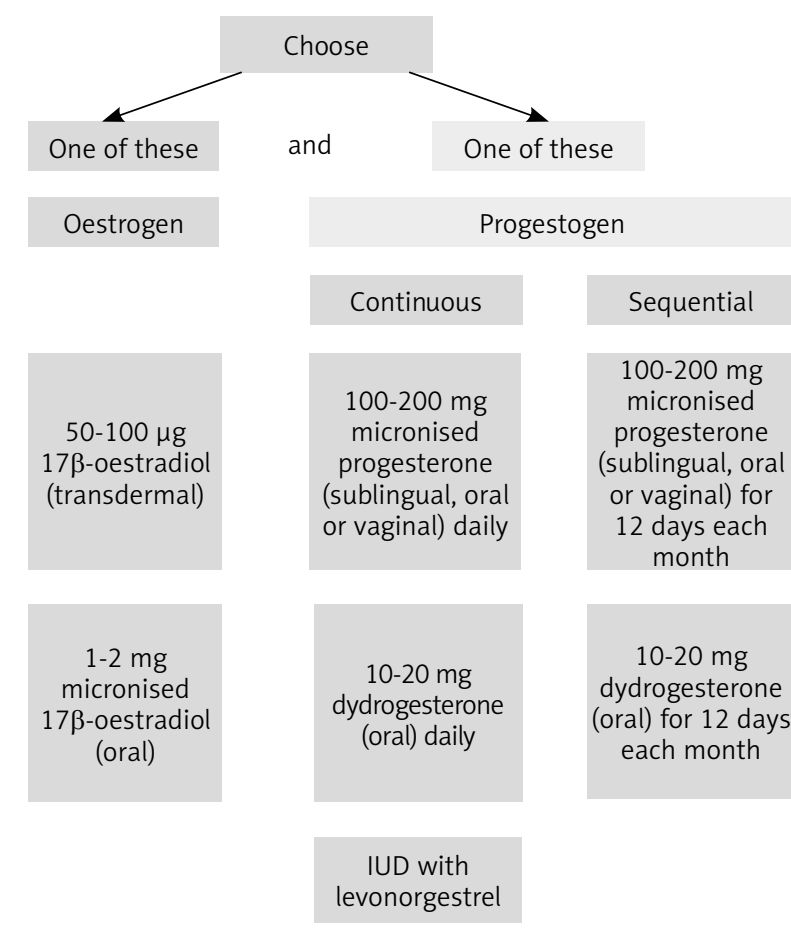

Fig. 1. Combined hormonal replacement therapy

\section{Hormonal replacement therapy}

Functional evidence has accumulated showing that restoring oestrogen action increases $B M D$ in patients with POI. Hormonal treatment should provide hormonal balance and should mimic normal ovarian function as much as possible. Hormonal replacement therapy (HRT) should be prescribed to protect against long-term health consequences of hypoestrogenism to patients with $\mathrm{POI}$ without contraindications to systemic hormonal therapy. First-line hormonal therapy in women suffering from $\mathrm{POI}$ is combined HRT. The addition of appropriate doses of progestogen is crucial for protection against endometrial hyperplasia (Fig. 1). Doses of oestradiol should be appropriate for achieving good control of symptoms and protection against side health consequences; nevertheless, assessment of oestradiol concentration should not be conducted [21]. Patients suffering from POI without properly developed secondary sex characteristics and presenting primary amenorrhoea should be prescribed increasing doses of oestrogen replacement therapy due to gradual maturation induction. Progestogen should be added after six months to two years or when breakthrough bleeding begins [22].

Combined hormonal contraceptives can be prescribed to patients particularly seeking ovulation preservation, despite the fact that in POI patients ovulation is rare [21]. Insertion of an intrauterine device with levonorgestrel due to protection of endometrial hyperplasia and against pregnancy with additional administration of non-contraceptive oestrogens is noteworthy. This route of progestogen administration is associated with better compliance and higher protection against non-atypical and atypical hyperplasia. Moreover, intrauterine administration of levonorgestrel provides continuous protection of endometrium without complications associated with exposure to systemic progestogen. It is estimated that oral administration of progestogen decreases the oestrogenic protective effect on the cardiovascular system, and can induce metabolic changes and premenstrual mood changes, nausea, headaches, as well as mastalgia [23].

\section{Bisphosphonates}

Bisphosphonates, due to their inhibiting action on osteoclast activity, are well-established agents reducing resorption of BMD. Bisphosphonates bind to hydroxyapatite crystals and thus are selectively adsorbed to the bone mineral surface. They are preferentially incorporated into sites characterised by accelerated skeletal turnover, where active bone remodelling occurs. It is well established that they decrease the risk of vertebral fracture by $50-60 \%$ and non-vertebral fracture by 20-30\% [17]. Bisphosphonates must be used with caution in young patients (such as POI patients) because of 
their very long skeletal half-life and non-clarified influence on foetal development. Despite the fact that spontaneous pregnancies in $\mathrm{POI}$ patients are rare, they must be used with caution and should not be prescribed to young women, particularly those who might eventually conceive [24]. In the case of planned pregnancy, it is important to withdraw bisphosphonates for at least one year before fertilisation $[17,25]$.

\section{Selective oestrogen receptor modulators}

Raloxifene is a selective oestrogen receptor modulator (SERM) approved for long-term treatment of decreased BMD and osteoporotic fractures. It plays an activatory role on oestrogen receptor located in bone tissue. The decline of bone resorption is associated particularly with simultaneous double action: inhibition of osteoclastic activity by decreasing IL- 6 concentration, and induction of osteoblastic activity. It reduces the risk of vertebral fractures by $30-50 \%$ in postmenopausal women $[26,27]$. It seems that raloxifene does not influence the risk of non-vertebral fractures. Another SERM used in the treatment of osteoporosis is bazedoxifene, which significantly reduces the risk of new vertebral fractures in postmenopausal women with osteoporosis. Moreover, in a three-year active-controlled study it decreased the risk of non-vertebral fractures in patients with higher fracture risk [28].

\section{Other medical approaches}

Teriparatide is an alternative to osteoporosis treatment. Given by daily injection for up to two years, it reduces the risk of vertebral and non-vertebral fracture. Strontium ranelate can also be used in the treatment of decreased BMD in postmenopausal women. Notwithstanding the fact that the mechanism of action is unclear, it reduces both vertebral and non-vertebral fracture risk. Last but not least in osteoporosis treatment is denosumab, a receptor activator of RANKL-inhibitor, which improves BMD and prevents vertebral and non-vertebral fractures. An additional benefit of denosumab is good compliance with the patient because it is administered subcutaneously only once per six months [17].

\section{Assessment of treatment}

Treatment-induced increase in BMD with inhibitors of bone turnover is modest (typically $2 \%$ per year). Taking into consideration that the precision error of DEXA in repeated measurements is estimated at $1-2 \%$, the time interval of repeat estimates must be long enough to achieve changes in BMD. Otherwise the differences in BMD may result from errors in the DEXA measurements, not from BMD improvement [29, 30]. Studies indicate that if postmenopausal patients do not suffer from any additional diseases, a five-year interval between DEXA measurements may be appropriate.

\section{Disclosure}

The authors report no conflict of interest.

\section{References}

1. Manolagas SC, O'Brien CA, Almeida M. The role of estrogen and androgen receptors in bone health and disease. Nat Rev Endocrinol 2013; 9: 699712.

2. Manolagas SC. Birth and Death of Bone Cells: Basic Regulatory Mechanisms and Implications for the Pathogenesis and Treatment of Osteoporosis. Endocr Rev 2000; 21: 115-137.

3. Almeida M, Han L, Martin-Millan M, et al. Skeletal Involution by Age-associated Oxidative Stress and Its Acceleration by Loss of Sex Steroids. J Biol Chem 2007; 282: 27285-27297.

4. Parfitt AM, Villanueva AR, Foldes J, Rao DS. Relations between histologic indices of bone formation: Implications for the pathogenesis of spinal osteoporosis. J Bone Miner Res 2009; 10: 466-473.

5. Riggs BL, Khosla S, Melton LJ. A Unitary Model for Involutional Osteoporosis: Estrogen Deficiency Causes Both Type I and Type II Osteoporosis in Postmenopausal Women and Contributes to Bone Loss in Aging Men. J Bone Miner Res 1998; 13: 763-773.

6. Khosla S, Melton LJ, Atkinson EJ, et al. Relationship of Serum Sex Steroid Levels and Bone Turnover Markers with Bone Mineral Density in Men and Women: A Key Role for Bioavailable Estrogen 1. J Clin Endocrinol Metab 1998; 83: 2266-2274.

7. Han Z-H, Palnitkar S, Rao DS, et al. Effects of Ethnicity and Age or Menopause on the Remodeling and Turnover of Iliac Bone: Implications for Mechanisms of Bone Loss. J Bone Miner Res 1997; 12: 498-508.

8. Onal $M$, Xiong J, Chen X, et al. Receptor activator of nuclear factor $\kappa \mathrm{B}$ ligand (RANKL) protein expression by $\mathrm{B}$ lymphocytes contributes to ovariectomy-induced bone loss. J Biol Chem 2012; 287: 29851-29860.

9. Leite-Silva P, Bedone A, Pinto-Neto AM, et al. Factors associated with bone density in young women with karyotypically normal spontaneous premature ovarian failure. Arch Gynecol Obstet 2009; 280: 177-181.

10. Freriks K, Timmermans J, Beerendonk CCM, et al. Standardized Multidisciplinary Evaluation Yields Significant Previously Undiagnosed Morbidity in Adult Women with Turner Syndrome. J Clin Endocrinol Metab 2011; 96: E1517-E1526.

11. Popat VB, Calis KA, Kalantaridou SN, et al. Bone mineral density in young women with primary ovarian insufficiency: results of a three-year randomized controlled trial of physiological transdermal estradiol and testosterone replacement. J Clin Endocrinol Metab 2014; 99: 3418-3426.

12. Ratcliffe MA, Lanham SA, Reid DM, Dawson AA. Bone mineral density $(B M D)$ in patients with lymphoma: the effects of chemotherapy, intermittent corticosteroids and premature menopause. Hematol Oncol 1992; 10: 181-187.

13. Anasti JN, Kalantaridou SN, Kimzey LM, et al. Bone loss in young women with karyotypically normal spontaneous premature ovarian failure. Obstet Gynecol 1998; 91: 12-15.

14. Popat VB, Calis KA, Vanderhoof VH, et al. Bone Mineral Density in Estrogen-Deficient Young Women. J Clin Endocrinol Metab 2009; 94: $2277-$ 2283.

15. Uygur D, Sengül Ö, Bayar D, et al. Bone loss in young women with premature ovarian failure. Arch Gynecol Obstet 2005; 273: 17-19.

16. Stavraka C, Maclaran K, Gabra H, et al. A study to evaluate the cause of bone demineralization in gynecological cancer survivors. Oncologist 2013; 18: 423-429.

17. European Society for Human Reproduction and Embryology (ESHRE) Guideline Group on POI, Webber L, Davies M, et al. ESHRE Guideline: management of women with premature ovarian insufficiency. Hum Reprod 2016; 31: 926-937.

18. Giraldo H, Benetti-Pinto C, Ferreira V, et al. Standard hormone therapy is inadequate for bone density in premature ovarian insufficiency. Gynecol Endocrinol 2017; 33: 283-286. 
19. Moayyeri A, Adams JE, Adler RA, et al. Quantitative ultrasound of the heel and fracture risk assessment: an updated meta-analysis. Osteoporos Int 2012; 23: 143-153.

20. Cosman F, de Beur SJ, LeBoff MS, et al. Clinician's Guide to Prevention and Treatment of Osteoporosis. Osteoporos Int 2014; 25: 2359-2381.

21. Committee on Gynecologic Practice. Committee Opinion No. 698. Obstet Gynecol 2017; 129: e134-e141.

22. Baker VL. Primary ovarian insufficiency in the adolescent. Curr Opin Obstet Gynecol 2013; 25: 375-381.

23. Wildemeersch $D$. Why perimenopausal women should consider to use a levonorgestrel intrauterine system. Gynecol Endocrinol 2016; 32: 659661.

24. Marino R, Misra M. Bone Health in Primary Ovarian Insufficiency. Semin Reprod Med 2011; 29: 317-327.

25. Drake MT, Clarke BL, Khosla S. Bisphosphonates: mechanism of action and role in clinical practice. Mayo Clin Proc 2008; 83: 1032-1045.

26. Gizzo S, Saccardi C, Patrelli TS, et al. Update on Raloxifene. Obstet Gynecol Surv 2013; 68: 467-481.

27. Nagatoya K, Nishimoto K, Shibahara N, et al. Effects of raloxifene on bone metabolism in postmenopausal women on chronic hemodialysis. Clin Exp Nephrol 2015; 19: 939-946.

28. Silverman SL, Christiansen C, Genant HK, et al. Efficacy of Bazedoxifene in Reducing New Vertebral Fracture Risk in Postmenopausal Women With Osteoporosis: Results From a 3-Year, Randomized, Placebo-, and Active-Controlled Clinical Trial. J Bone Miner Res 2008; 23: 1923-1934.

29. Olivares JL, Ortiz VA, Mayer M, et al. [An approach to a sanitary and social problem: urinary iodine excretion in pregnant women from a iodine deficient region]. Arch Latinoam Nutr 2009; 59: 378-382.

30. Baim S, Wilson CR, Lewiecki EM, et al. Precision assessment and radiation safety for dual-energy $\mathrm{X}$-ray absorptiometry: position paper of the International Society for Clinical Densitometry. J Clin Densitom 2005; 8: 371-378. 